\title{
Investigation of an Idiopathic Lesion in Redclaw Crayfish Cherax Quadricarinatus Using Suppression Subtractive Hybridization
}

\author{
Rusaini $^{1,2}$, Ellen Ariel ${ }^{2}$, Graham W. Burgess ${ }^{2}$ and Leigh Owens ${ }^{2}$ \\ ${ }^{1}$ Aquaculture, Faculty of Agriculture, University of Tadulako, Bumi Tadulako Tondo, Palu, Indonesia \\ ${ }^{2}$ Microbiology and Immunology, School of Veterinary and Biomedical Sciences, James Cook University, \\ Townsville, Australia
}

Correspondence should be addressed to: Rusaini; rusaini.rusaini@my.jcu.edu.au, rmallullu@yahoo.com

Received 18 January 2013; Accepted 27 February 2013; Published 24 April 2013

Academic Editor: Parin Chaivisuthangkura

Copyright @ 2013 Rusaini, Ellen Ariel, Graham W. Burgess and Leigh Owens. Distributed under Creative Commons CC-BY 3.0

\begin{abstract}
Lesions indicative of putative parvovirus infection were detected in a population of freshwater redclaw crayfish (Cherax quadricarinatus) displaying hypertrophied nuclei, while these changes were undetected in another population. These changes were characterised by hypertrophic nuclei with marginated chromatin but without Cowdry type A (CA) intranuclear inclusion bodies. Suppression subtractive hybridization (SSH) was performed to generate a cDNA forward library between the two populations of crayfish. A total of 323 sequences were analysed from SSH libraries. These sequences were grouped into 76 consensus sequences and clustered into 8 categories based on sequence homology from the NCBI GenBank database. Even though histopathological observations consistently revealed lesions presumptive of putative parvovirus in C. quadricarinatus as previously reported, the molecular method failed to confirm a viral aetiology. Despite the absence of viral gene detection and therefore a conclusion of an idiopathic aetiology, the health status of these two populations of redclaw crayfish was different, resulting in differentially expressed immune-related genes in the two populations, with some genes being up-regulated in the hypertrophied nuclei population. Furthermore, the absence of parvovirus from the SSH libraries indicates that perhaps the viral messenger RNA was in too low a concentration to be detected or does not have any poly(A) tail which the SSH methodology needs to function correctly.
\end{abstract}

Keywords: Redclaw crayfish, Parvovirus, Suppression subtractive hybridization, Idiopathic aetiology.

\section{Introduction}

Like their marine crustacean counterparts, freshwater redclaw crayfish (Cherax quadricarinatus) can be affected detrimentally by viral infections. In Australia alone, several viruses have been found to cause infection in redclaw crayfish. Cherax quadricarinatus bacilliform virus was reported to be widespread in wild and cultured redclaw crayfish in northern Queensland between 1992 and 1996 (Anderson and Prior, 1992; Edgerton, 1996; Edgerton and Owens, 1999). Cherax Giardiavirus-like virus was also commonly found in cultured redclaw crayfish and 
thought to be a significant pathogen of juveniles (Edgerton and Owens, 1997; Edgerton et al., 1994; Edgerton and Owens, 1999). In addition, a presumptive hepatopancreatic reovirus has been reported to decrease stress resistance in farmed C. quadricarinatus (Edgerton et al., 2000; Hayakijkosol and Owens, 2011). Even though these viruses are not very virulent to redclaw crayfish, they have been shown to cause low level mortalities and growth retardation and are thought to suppress the immunoresponse of animals (Edgerton and Owens, 1999; Hayakijkosol and Owens, 2011). These orphan viruses (viruses which infect a host, but are not considered to cause any diseases) have been ignored as causes of economic losses in cultured crayfish. However, when eggs specific-pathogen-free for these viruses were produced as hatchery technology developed, the culture period shortened by approximately $15 \%$ and production doubled, thereby reducing the production cost and increasing the profit margin (Owens, 2011).

Another disease caused by spawner-isolated mortality virus (SMV) was previously detected in penaeid prawns, but has since caused significant mortality in captured and transported redclaw crayfish in 1997 - 1998 (Owens and McElnea, 2000). Therefore, these authors predicted that viral diseases of penaeid prawns may have potential effects on the health status of crayfish. Moreover, white spot syndrome virus (WSSV), the main cause of catastrophic losses within the penaeid prawn industry, can also infect crayfish both experimentally and naturally in wild and farmed animals (Baumgartner et al., 2009; Edgerton, 2004; Edgerton et al., 2002; Jiravanichpaisal et al., 2001).

Putative parvovirus infection has been documented to be associated with chronic and mass mortalities in freshwater crayfish. Tissue changes and viral morphology have been described using light microscopy and transmission electron microscopy (TEM) (Bowater et al., 2002; Edgerton et al., 1997; Edgerton et al., 2000). However, genetic characterisation of the viruses has not been carried out and studies on immune-related genes of redclaw crayfish are sparse. Lesions characterised by hypertrophied nuclei with rarefied chromatin but without Cowdry type A (CA) intranuclear inclusion bodies that resemble those associated with putative parvovirus infection previously reported in $C$. quadricarinatus (Edgerton et al., 2000) were observed in a population, while these changes were not observed in another population of redclaw crayfish in the aquaculture facilities of the School of Veterinary and Biomedical Sciences, James Cook University. Therefore, this study was conducted to profile possible viral genomes and differentially expressed genes from gills of the two populations of crayfish using suppression subtractive hybridization (SSH).

\section{Materials and Methods}

\section{Experimental Animals}

Redclaw crayfish (Cherax quadricarinatus) were sampled from the aquaculture facilities at the School of Veterinary and Biomedical Sciences, James Cook University. One population which comprised about 200 animals was maintained in an outdoor facility (hypertrophied nuclei population) while the other population which consist of about 500 animals was in an indoor facility (nonhypertrophied nuclei population). Both populations were reared in 1,000 l plastic bins with a recirculating system. Experimental animals were anaesthetised by placing them in iced water for a few minutes prior to gill excision and histological preparation.

\section{Histology}

The cephalothorax was fixed in Davidson's fixative (formaldehyde $220 \mathrm{ml}$, acetic acid $115 \mathrm{ml}$, absolute ethanol $313 \mathrm{ml}$ and tap water $352 \mathrm{ml}$ ) at a ratio of tissue to fixative 1:10 for 24 hours. The cephalothorax was cut longitudinally, placed in a histocassette, stored in $70 \%$ ethanol and then processed for routine histological examination using standard paraffin embedded procedure (Bell and Lightner, 1988). Sections were cut at 5 $\mu \mathrm{m}$ using a rotary microtome, mounted on glass slides, stained with haematoxylin and eosin (H\& E) examined under a light microscopy.

\section{RNA Extraction}

Total RNA was extracted separately from the gills of hypertrophied nuclei (6 crayfish) and 
non-hypertrophied nuclei (6 crayfish) $C$. quadricarinatus using SV Total RNA Isolation System (Promega, USA) according to the manufacturer's instructions. The concentration and purity of total RNA was determined using spectrophotometry (NanoPhotometerTM, Implen, Germany) and stored at $-80^{\circ} \mathrm{C}$ until required. Polyadenylated (Poly(A)+) RNA was isolated from pooled total RNA using Poly A Tract mRNA Isolation System III (Promega, USA) following the manufacturer's protocol. Before use, $750 \mu \mathrm{l}$ Poly(A) ${ }^{+}$RNA was concentrated by freeze drying (Telstar 23750 - Cryodos -50/230 V 50 Hz, United Kingdom) and re-dissolved in $25 \mu \mathrm{l}$ RNase-free water. Concentration and purity of Poly (A) + RNA was examined using spectrophotometry.

\section{Suppression Subtractive Hybridization}

Suppression subtractive hybridization was performed using the PCR-Select cDNA Subtraction Kit (Clontech, USA) following the manufacturer's instructions to generate a cDNA forward library between hypertrophied nuclei population (tester) and nonhypertrophied nuclei population (driver). Briefly, the tester cDNA was prepared from $1.24 \mu \mathrm{g}$ of poly $(\mathrm{A})^{+}$RNA and the driver cDNA was synthesised from $1.16 \mu \mathrm{g}$ of poly(A)+ RNA. Both tester and driver cDNAs were digested with a four-base cutting restriction enzyme (Rsa I) to obtain blunt-ended molecules. Following this, tester cDNA was equally divided into two samples and ligated with one of the two different adaptors (adaptor 1 and adaptor 2R) to the 5' end of cDNA. The ligation efficiency was evaluated on a $1.2 \%$ agarose-TAE gel containing ethidium bromide (EtBr) at a concentration of $0.5 \mu \mathrm{g} / \mathrm{ml}$. Next, tester cDNAs were hybridized with an excess of driver cDNA at $68^{\circ} \mathrm{C}$ for 8 hours following denaturation at $98^{\circ} \mathrm{C}$ for 1.5 minutes. Two samples from the first hybridization were mixed and hybridized with an excess of freshly denatured driver cDNA overnight at $68^{\circ} \mathrm{C}$ to enrich the differentially expressed gene fraction. Finally, the mixture was subjected to two rounds of PCR using specific primers to both adaptors to amplify exponentially the target differentially expressed genes and suppress the common sequence of the two cDNA populations. These PCR products were examined on a $1.2 \%$ agarose/ethidium bromide gel.

\section{Cloning and Sequencing}

Subtracted PCR products were cloned into T \& A cloning vectors (RBC, Taiwan) or pGEM-T easy vectors (Promega, USA) and transformed into HIT-DH5 $\alpha$ or JM109 competent cells which were plated onto agar containing ampicillin, X-gal (5-bromo-4chloro-3-indolyl- $\beta$-D-galactopyranoside) and IPTG (isopropyl- $\beta$-D-thiogalactopyranoside). Plasmid DNA was extracted using Wizard ${ }^{\circledR}$ Plus SV Minipreps DNA Purification System (Promega, USA) from randomly selected white colonies and sent to Macrogen, Korea for sequencing. Nucleotide sequences were analysed with BLASTx and BLASTn against known amino acid/nucleotide sequences on GenBank databases (NCBI). Sequences with E-values $<1 \mathrm{e}^{-05}$ were considered significant.

\section{Results}

\section{Histology}

Lesions typical of putative parvovirus infection, consisting of hypertrophic nuclei with rarefied chromatin but without Cowdry type A inclusion body in the gill tissues (Fig. 1a) were found in the population displaying hypertrophied nuclei (10 out of 10 crayfish), while these lesions were not observed in the non-hypertrophied nuclei population (7/7) of crayfish (Fig. 1b). In the gills of the hypertrophied nuclei population, pyknotic and karyorrectic nuclei were also detected. Aggregation of haemocytes and granulomatous reactions in the gill tissues were seen in both populations suggesting the probability of subclinical bacterial infections. 

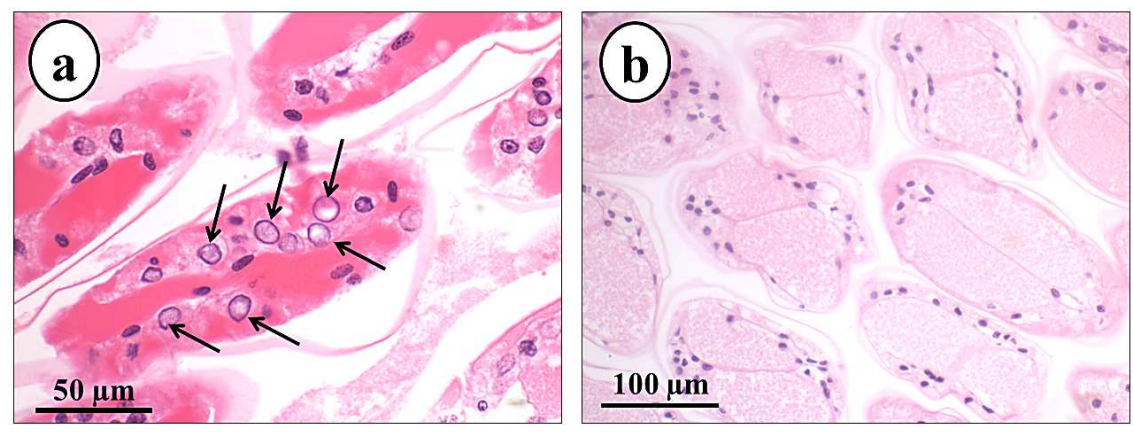

Fig. 1. Longitudinal Section of the Gills of Cherax Quadricarinatus from Hypertrophied Nuclei Population (a) and Non-hypertrophied Nuclei Population (b). Note Hypertrophy of Nuclei with arefied Chromatin (Arrow) in the Gills from the Hypertrophied Nuclei Population (a) Compared to Normal Gill Tissue from the Non-hypertrophied Nuclei Population (b)

\section{Haematoxylin and Eosin Stain}

\section{Suppression Subtractive Hybridization}

Suppression subtractive hybridization was conducted three times resulting in several libraries (Table 1). A total of 339 clones were sequenced. After removing vector sequences and the poor quality sequences of these three attempts, a total of 323 sequences were grouped into 76 consensus sequences (contigs) with a range of insert sizes between $61 \mathrm{bp}$ and $484 \mathrm{bp}$. The homology search revealed that around $61.6 \%$ of the total clones (199 out of 323 clones) shared significant similarities to known proteins in the GenBank database (Table 2).

Table 1. Gill cDNA Libraries Obtained from Approximate PCR Product Amplicons of Suppression Subtractive Hybridization (SSH) Trials

\begin{tabular}{|lll|}
\hline Trial & Libraries & PCR products \\
\hline \multirow{2}{*}{1} & $1 \mathrm{a}$ & $270 \mathrm{bp}$ \\
\cline { 2 - 3 } & $1 \mathrm{~b}$ & $200 \mathrm{bp}$ \\
\hline 2 & $2 \mathrm{a}$ & $200 \mathrm{bp}$ \\
\hline \multirow{3}{*}{3} & $3 \mathrm{a}$ & $450 \mathrm{bp}$ \\
\cline { 2 - 3 } & $3 \mathrm{~b}$ & $350 \mathrm{bp}$ \\
\cline { 2 - 3 } & $3 \mathrm{c}$ & $300 \mathrm{bp}$ \\
\hline
\end{tabular}

These transcripts were clustered into 8 categories based on sequence homology from the public database (Fig. 2). Significantly matching transcripts were clustered to immune-related genes (15.2\%), energy and metabolism factor genes $(3.1 \%)$ and muscle and cytoskeletal-related proteins (0.6\%). Transcripts that had significant similarity to amino acids of unknown functionalities in the public database were grouped into ribosomal $(2.8 \%)$ and hypothetical protein sequences
(39.9\%). These were the most abundant transcripts found in the SHH libraries. Transcripts that did not match any protein sequences but had significant matches with nucleotides in GenBank were clustered into redclaw crayfish mRNA (13.0\%) and other sequences $(0.6 \%)$. Sequences that had no significant matches either with amino acids or nucleotides in the public database were grouped into unknown sequences (24.8\%). 


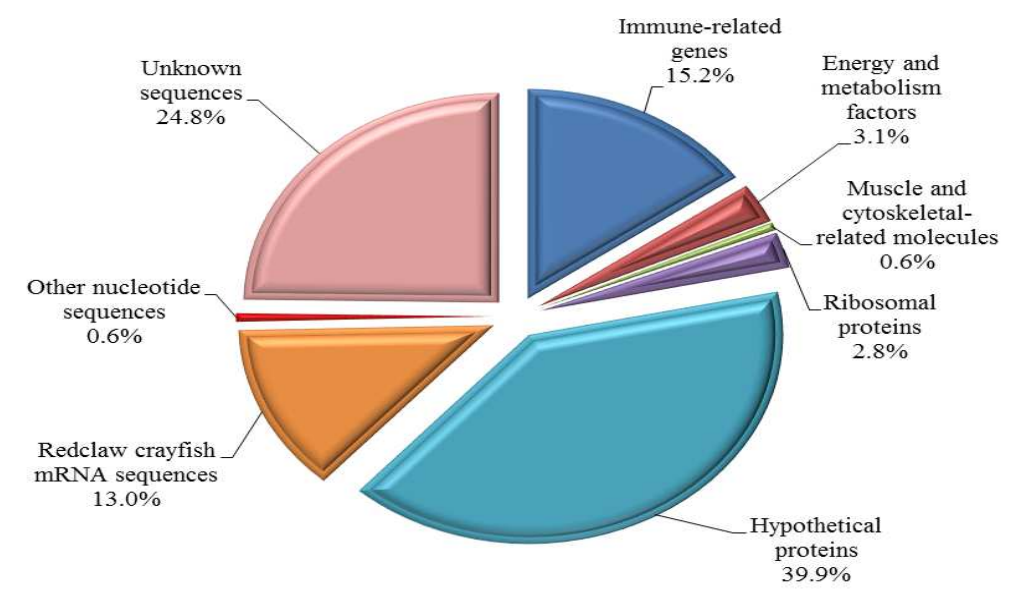

Fig. 2. Functional Categories of Differentially Expressed Genes From The Gill Cdna Suppression Subtractive Hybridization (SSH) Libraries Of Redclaw Crayfish Cherax Quadricarinatus

Table 2. Differentially Expressed Genes from Suppression Subtractive Hybridization (SSH) Libraries of the Gills of Freshwater Redclaw Crayfish, Cherax Quadricarinatus with Amino Acids/Sequences Similarity to Amino Acids/Sequences in the GenBank Database (NCBI)

\begin{tabular}{|c|c|c|c|c|c|c|c|c|c|}
\hline Contig & $\begin{array}{l}\text { Number } \\
\text { of clones }\end{array}$ & Library & $\begin{array}{l}\text { Fragment } \\
\text { size (bp) }\end{array}$ & $\begin{array}{l}\text { BLAST } \\
\text { type }\end{array}$ & $\begin{array}{l}\text { Accession } \\
\text { number }\end{array}$ & Closest homology & Species & E-value & $\begin{array}{l}\text { Identity (\%) - } \\
\text { (q/s) }\end{array}$ \\
\hline \multicolumn{6}{|l|}{ 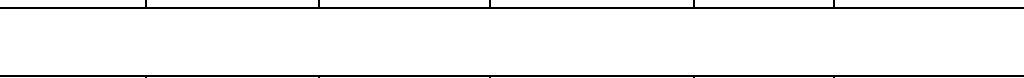 } & \multicolumn{4}{|l|}{$\begin{array}{l}\text { Immune-related } \\
\text { genes }\end{array}$} \\
\hline CqG003 & 1 & 3 & 302 & $\mathrm{X}$ & ABC59529.1 & $\begin{array}{l}\text { Cytosolic manganese } \\
\text { superoxide dismutase }\end{array}$ & $\begin{array}{l}\text { Penaeus } \\
\text { vannamei }\end{array}$ & $2.00 \mathrm{E}-09$ & $93(26 / 28)$ \\
\hline CqG008 & 6 & $3 a$ & 360 & $\mathrm{X}$ & ACD76641.1 & C-type lysozyme & $\begin{array}{l}\text { Penaeus } \\
\text { stylirostris }\end{array}$ & $3.00 \mathrm{E}-08$ & $63(24 / 38)$ \\
\hline CqG015 & 17 & $\begin{array}{l}3,3 a, 3 b, \\
3 c\end{array}$ & 235 & $\mathrm{X}$ & P19857.2 & $\begin{array}{lll}\begin{array}{l}\text { Serum } \\
\text { protein }\end{array} & \text { amyloid } & \mathrm{A} \\
\end{array}$ & Equus cabalus & $9.00 \mathrm{E}-06$ & $76(22 / 29)$ \\
\hline CqG018 & 1 & $3 c$ & 243 & $\mathrm{X}$ & ACL79888.1 & Putative elastin a & $\begin{array}{l}\text { Rimicaris } \\
\text { exoculata }\end{array}$ & $1.00 \mathrm{E}-22$ & $75(41 / 55)$ \\
\hline CqG025 & 1 & $3 c$ & 240 & $\mathrm{X}$ & ACY66442.1 & $\begin{array}{l}\text { Eukaryotic initiation } \\
\text { factor } 4 \mathrm{~A}\end{array}$ & $\begin{array}{l}\text { Scylla } \\
\text { paramamosain }\end{array}$ & $7.00 \mathrm{E}-39$ & $99(79 / 80)$ \\
\hline CqG026 & 1 & $3 c$ & 150 & $\mathrm{X}$ & ACY66461.1 & $\begin{array}{l}\text { Translationally- } \\
\text { controlled tumour } \\
\text { protein }\end{array}$ & $\begin{array}{l}\text { Scylla } \\
\text { paramamosain }\end{array}$ & $1.00 \mathrm{E}-18$ & $78(38 / 49)$ \\
\hline CqG027 & 1 & $3 c$ & 161 & $\mathrm{X}$ & ACY66388.1 & Chaperonin 10 & $\begin{array}{l}\text { Scylla } \\
\text { paramamosain }\end{array}$ & $3.00 \mathrm{E}-14$ & $85(45 / 53)$ \\
\hline CqG029 & 1 & $3 c$ & 237 & $\mathrm{X}$ & ABZ90154.1 & $\begin{array}{l}\text { Translationally- } \\
\text { controlled tumor } \\
\text { protein }\end{array}$ & Penaeus japonicus & $1.00 \mathrm{E}-29$ & $86(49 / 57)$ \\
\hline CqG030 & 2 & $3 a, 3 c$ & 368 & $\mathrm{X}$ & ACY64752.1 & Crustin 2 & $\begin{array}{l}\text { Procambarus } \\
\text { clarkii }\end{array}$ & $4.00 \mathrm{E}-49$ & $74(64 / 87)$ \\
\hline CqG047 & 1 & 3 & 388 & $\mathrm{X}$ & AEL23029.1 & $\begin{array}{l}\text { Insulin-like growth } \\
\text { factor binding protein } \\
\text { 7-like protein }\end{array}$ & $\begin{array}{l}\text { Cherax } \\
\text { quadricarinatus }\end{array}$ & $2.00 \mathrm{E}-37$ & $96(54 / 56)$ \\
\hline CqG048 & 14 & $\begin{array}{l}2 \mathrm{a}, 3,3 \mathrm{a}, \\
3 \mathrm{c}\end{array}$ & 343 & $\mathrm{X}$ & ADI96221.1 & $\begin{array}{l}\text { Kazal-type serine } \\
\text { proteinase inhibitor I }\end{array}$ & $\begin{array}{l}\text { Procambarus } \\
\text { clarkii }\end{array}$ & $2.00 \mathrm{E}-17$ & $76(34 / 45)$ \\
\hline CqG050 & 2 & $3,3 b$ & 269 & $\mathrm{X}$ & ABH10628.1 & Laminin receptor & $\begin{array}{l}\text { Penaeus } \\
\text { vannamei }\end{array}$ & $1.00 \mathrm{E}-38$ & $92(79 / 86)$ \\
\hline CqG075 & 1 & $3 a$ & 388 & $\mathrm{X}$ & ADM21460.1 & $\begin{array}{l}\text { Anti- } \\
\text { lipopolysaccharide } \\
\text { factor (ALF) isoform } 6\end{array}$ & Penaeus monodon & $1.00 \mathrm{E}-43$ & $71(62 / 87)$ \\
\hline & & & & & & $\begin{array}{l}\text { Energy and } \\
\text { metabolism factors }\end{array}$ & & & \\
\hline CqG007 & 1 & 3 & 189 & $\mathrm{X}$ & YP_022769.1 & $\begin{array}{l}\text { NADH dehydrogenase } \\
\text { subunit } 3\end{array}$ & Cherax destructor & $2.00 \mathrm{E}-05$ & $67(33 / 49)$ \\
\hline CqG021 & 1 & $3 c$ & 215 & $\mathrm{X}$ & AAM11778.1 & $\begin{array}{l}\text { Cytochrome oxidase } \\
\text { subunit I }\end{array}$ & $\begin{array}{l}\text { Engaeus } \\
\text { strictifrons }\end{array}$ & $1.00 \mathrm{E}-36$ & $89(63 / 71)$ \\
\hline
\end{tabular}




\begin{tabular}{|c|c|c|c|c|c|c|c|c|c|}
\hline CqG024 & 4 & $2 a, 3,3 c$ & 254 & $\mathrm{X}$ & YP_022768.1 & $\begin{array}{l}\text { Cytochrome c oxidase } \\
\text { subunit III (COIII) }\end{array}$ & Cherax destructor & $2.00 \mathrm{E}-35$ & $78(62 / 79)$ \\
\hline CqG035 & 1 & 3 & 350 & $\mathrm{X}$ & CBW54880.1 & $\begin{array}{l}\text { Putative DEAD box } \\
\text { ATP-dependent RNA } \\
\text { helicase }\end{array}$ & Cancer pagurus & $1.00 \mathrm{E}-17$ & $95(37 / 39)$ \\
\hline CqG041 & 1 & 3 & 225 & $\mathrm{X}$ & ACR54103.1 & $\begin{array}{l}\text { ATP synthase subunit } \\
\mathrm{g}\end{array}$ & $\begin{array}{l}\text { Palaemonetes } \\
\text { varians }\end{array}$ & $2.00 \mathrm{E}-20$ & $79(37 / 47)$ \\
\hline CqG056 & 1 & $1 \mathrm{a}$ & 81 & $\mathrm{X}$ & $\begin{array}{l}\text { YP_004563978 } \\
.1\end{array}$ & $\begin{array}{l}\text { NADH dehydrogenase } \\
\text { subunit } 4\end{array}$ & $\begin{array}{l}\text { Homarus } \\
\text { americanus }\end{array}$ & $1.00 \mathrm{E}-06$ & $81(21 / 26)$ \\
\hline CqG076 & 1 & $3 a$ & 175 & $\mathrm{X}$ & YP_022765.1 & $\begin{array}{l}\text { Cytochrome c oxidase } \\
\text { subunit II (COII) }\end{array}$ & Cherax destructor & $5.00 \mathrm{E}-15$ & $89(32 / 36)$ \\
\hline \multirow[t]{2}{*}{ Contig } & $\begin{array}{l}\text { Number } \\
\text { of clones }\end{array}$ & Library & $\begin{array}{l}\text { Fragment } \\
\text { size (bp) }\end{array}$ & $\begin{array}{l}\text { BLAST } \\
\text { type }\end{array}$ & $\begin{array}{l}\text { Accession } \\
\text { number }\end{array}$ & Closest homology & Species & E-value & $\begin{array}{l}\text { Identity (\%) - } \\
\text { (q/s) }\end{array}$ \\
\hline & & & & & & $\begin{array}{l}\text { Muscle/cytoskeletal } \\
\text { related-molecules }\end{array}$ & & & \\
\hline CqG037 & 1 & 2 & 76 & $\mathrm{X}$ & AAS98886.1 & Allergen Pen m2 & Penaeus chinensis & $2.00 \mathrm{E}-10$ & $100(25 / 25)$ \\
\hline CqG060 & 1 & $3 a$ & 405 & $\mathrm{X}$ & BAJ14323.1 & Alpha tubulin & Pinctada fucata & $1.00 \mathrm{E}-66$ & $96(96 / 100)$ \\
\hline \multicolumn{6}{|l|}{490000} & Ribosomal proteins & & & \\
\hline CqG014 & 1 & $3 c$ & 240 & $\mathrm{X}$ & AEB54647.1 & $\begin{array}{l}\text { Ribosomal protein } \\
\text { S18 }\end{array}$ & $\begin{array}{l}\text { Procambarus } \\
\text { clarkii }\end{array}$ & $2.00 \mathrm{E}-33$ & $100(76 / 76)$ \\
\hline CqG022 & 1 & $3 c$ & 265 & $\mathrm{X}$ & ADY39535.1 & $\begin{array}{l}\text { Putative } 60 \mathrm{~S} \\
\text { ribosomal protein } \mathrm{L7}- \\
\text { like }\end{array}$ & $\begin{array}{l}\text { Hottentotta } \\
\text { judaicus }\end{array}$ & $1.00 \mathrm{E}-41$ & $75(61 / 81)$ \\
\hline CqG058 & 2 & $2,2 a$ & 62 & $\mathrm{X}$ & ACY66551.1 & $\begin{array}{ll}\text { Ribosomal protein } \\
\text { L10 }\end{array}$ & $\begin{array}{l}\text { Scylla } \\
\text { paramamosain }\end{array}$ & $2.00 \mathrm{E}-05$ & $95(19 / 20)$ \\
\hline CqG062 & 2 & $3 \mathrm{~b}$ & 191 & $\mathrm{X}$ & $\begin{array}{l}\text { XP_002733250 } \\
.1\end{array}$ & $\begin{array}{ll}\text { PREDICTED: } \\
\text { Ribosomal } \\
\text { L38-like }\end{array}$ & $\begin{array}{l}\text { Saccoglossus } \\
\text { kowalevskii }\end{array}$ & $6.00 \mathrm{E}-20$ & $95(40 / 42)$ \\
\hline CqG064 & 2 & $3 b, 3 c$ & 275 & $\mathrm{X}$ & ADW95789.1 & $\begin{array}{l}\text { Ribosomal protein } \\
\text { S30-like protein }\end{array}$ & Pectinaria gouldii & $3.00 \mathrm{E}-11$ & $51(31 / 61)$ \\
\hline \multirow[t]{2}{*}{ CqG070 } & 1 & $3 c$ & 138 & $\mathrm{X}$ & ACN44179.1 & $\begin{array}{ll}\text { Ribosomal protein } \\
\text { S16 }\end{array}$ & Cavia porcellus & $2.00 \mathrm{E}-17$ & $89(50 / 56)$ \\
\hline & & & & & & $\begin{array}{l}\text { Hypothetical } \\
\text { proteins }\end{array}$ & & & \\
\hline CqG023 & 4 & $3 b, 3 c$ & 229 & $\mathrm{X}$ & DAA34691.1 & $\begin{array}{l}\text { TPA_inf: hypothetical } \\
\text { secreted protein } 323\end{array}$ & $\begin{array}{l}\text { Amblyomma } \\
\text { variegatum }\end{array}$ & $2.00 \mathrm{E}-11$ & $39(30 / 77)$ \\
\hline CqG028 & 121 & $\begin{array}{l}1,1 a, 1 b, 2, \\
2 a, 3,3 a, \\
3 b, 3 c\end{array}$ & 437 & $\mathrm{X}$ & CAM36311.1 & Hypothetical protein & $\begin{array}{l}\text { Thermobia } \\
\text { domestica }\end{array}$ & $8.00 \mathrm{E}-07$ & $71(23 / 32)$ \\
\hline CqG065 & 1 & $3 \mathrm{~b}$ & 305 & $\mathrm{X}$ & EFZ23151.1 & $\begin{array}{ll}\text { Hypothetical protein } \\
\text { SINV_03072 }\end{array}$ & Solenopsis invicta & $3.00 \mathrm{E}-20$ & $62(61 / 98)$ \\
\hline CqG066 & 1 & $3 \mathrm{~b}$ & 155 & $\mathrm{X}$ & $\begin{array}{l}\text { XP_002739723 } \\
.1\end{array}$ & $\begin{array}{l}\text { PREDICTED: Protein- } \\
\text { like }\end{array}$ & $\begin{array}{l}\text { Saccoglossus } \\
\text { kowalevskii }\end{array}$ & $1.00 \mathrm{E}-15$ & $87(34 / 39)$ \\
\hline CqG068 & 1 & $3 \mathrm{~b}$ & 240 & $\mathrm{X}$ & EFX85348.1 & $\begin{array}{l}\text { Hypothetical protein } \\
\text { DAPPUDRAFT_23054 } \\
5\end{array}$ & Daphnia pulex & $5.00 \mathrm{E}-22$ & $68(54 / 79)$ \\
\hline \multirow[t]{2}{*}{ CqG073 } & 1 & $3 c$ & 166 & $\mathrm{X}$ & XP_780871.2 & $\begin{array}{l}\text { PREDICTED: } \\
\text { Hypothetical protein }\end{array}$ & $\begin{array}{l}\text { Strongylocentratu } \\
\text { s purpuratus }\end{array}$ & $1.00 \mathrm{E}-06$ & $43(23 / 53)$ \\
\hline & & & & & & $\begin{array}{l}\text { Redclaw crayfish } \\
\text { mRNA sequences }\end{array}$ & & & \\
\hline CqG002 & 21 & $\begin{array}{l}1,1 a, 1 b, 2, \\
2 a\end{array}$ & 61 & n (h) & EF692627.1 & $\begin{array}{l}\text { Clone y9_B8 mRNA } \\
\text { sequences }\end{array}$ & $\begin{array}{l}\text { Cherax } \\
\text { quadricarinatus }\end{array}$ & $3.00 \mathrm{E}-21$ & $98(60 / 61)$ \\
\hline CqG005 & 3 & $1 b, 2 a, 3 c$ & 86 & n (h) & GQ286092.1 & $\begin{array}{l}\text { Clone GB_1A mRNA } \\
\text { sequences }\end{array}$ & $\begin{array}{l}\text { Cherax } \\
\text { quadricarinatus }\end{array}$ & $1.00 \mathrm{E}-27$ & $93(82 / 88)$ \\
\hline CqG012 & 1 & $3 \mathrm{~b}$ & 233 & n (h) & DQ847728.1 & $\begin{array}{l}\text { Clone cherax_207 } \\
\text { mRNA sequences }\end{array}$ & $\begin{array}{l}\text { Cherax } \\
\text { quadricarinatus }\end{array}$ & $1.00 \mathrm{E}-67$ & $\begin{array}{l}100 \\
(143 / 143)\end{array}$ \\
\hline CqG016 & 1 & $3 c$ & 170 & n (h) & DQ847803.1 & $\begin{array}{l}\text { Clone y1_a2 mRNA } \\
\text { sequences }\end{array}$ & $\begin{array}{l}\text { Cherax } \\
\text { quadricarinatus }\end{array}$ & $7.00 \mathrm{E}-78$ & $99(163 / 164)$ \\
\hline CqG020 & 2 & $3,3 c$ & 242 & n (h) & DQ847728.1 & $\begin{array}{l}\text { Clone } \quad \text { cherax_207 } \\
\text { mRNA sequences }\end{array}$ & $\begin{array}{l}\text { Cherax } \\
\text { quadricarinatus }\end{array}$ & $3.00 \mathrm{E}-98$ & $99(203 / 205)$ \\
\hline Contig & $\begin{array}{l}\text { Number } \\
\text { of clones }\end{array}$ & Library & $\begin{array}{l}\text { Fragment } \\
\text { size (bp) }\end{array}$ & $\begin{array}{l}\text { BLAST } \\
\text { type }\end{array}$ & $\begin{array}{l}\text { Accession } \\
\text { number }\end{array}$ & Closest homology & Species & E-value & $\begin{array}{l}\text { Identity (\%) - } \\
\text { (q/s) }\end{array}$ \\
\hline CqG031 & 2 & $3,3 c$ & 221 & $\mathrm{n}(\mathrm{h})$ & DQ847679.1 & $\begin{array}{l}\text { Clone cherax_157 } \\
\text { mRNA sequences }\end{array}$ & $\begin{array}{l}\text { Cherax } \\
\text { quadricarinatus }\end{array}$ & $1.00 \mathrm{E}-106$ & $99(220 / 223)$ \\
\hline CqG034 & 3 & 1,3 & 374 & n (h) & DQ847684.1 & $\begin{array}{l}\text { Clone cherax_163 } \\
\text { mRNA sequences }\end{array}$ & $\begin{array}{l}\text { Cherax } \\
\text { quadricarinatus }\end{array}$ & $7.00 \mathrm{E}-171$ & $99(333 / 335)$ \\
\hline CqG036 & 2 & $1,1 \mathrm{~b}$ & 73 & $\mathrm{n}(\mathrm{h})$ & DQ847743.1 & Clone cherax_223 & Cherax & $9.00 \mathrm{E}-28$ & $99(72 / 73)$ \\
\hline
\end{tabular}




\begin{tabular}{|c|c|c|c|c|c|c|c|c|c|}
\hline & & & & & & mRNA sequences & quadricarinatus & & \\
\hline CqG045 & 2 & 3 & 233 & $\mathrm{n}(\mathrm{h})$ & DQ847664.1 & $\begin{array}{l}\text { Clone cherax_141 } \\
\text { mRNA sequences }\end{array}$ & $\begin{array}{l}\text { Cherax } \\
\text { quadricarinatus }\end{array}$ & $6.00 \mathrm{E}-90$ & $96(200 / 208)$ \\
\hline CqG046 & 1 & 3 & 135 & $n(h)$ & DQ847565.1 & $\begin{array}{l}\text { Clone epi2_G11 mRNA } \\
\text { sequences }\end{array}$ & $\begin{array}{l}\text { Cherax } \\
\text { quadricarinatus }\end{array}$ & $2.00 \mathrm{E}-38$ & $98(94 / 96)$ \\
\hline CqG049 & 1 & 3 & 260 & n (h) & EF692615.1 & $\begin{array}{l}\text { Clone y17_B11 mRNA } \\
\text { sequences }\end{array}$ & $\begin{array}{l}\text { Cherax } \\
\text { quadricarinatus }\end{array}$ & $1.00 \mathrm{E}-132$ & $\begin{array}{l}100 \\
(260 / 260)\end{array}$ \\
\hline CqG051 & 1 & 3 & 484 & n (h) & DQ847548.1 & $\begin{array}{l}\begin{array}{l}\text { Clone epi1_B3 mRNA } \\
\text { sequences }\end{array} \\
\text { sequent }\end{array}$ & $\begin{array}{l}\text { Cherax } \\
\text { quadricarinatus }\end{array}$ & $0.00 \mathrm{E}+00$ & $99(464 / 467)$ \\
\hline \multirow[t]{2}{*}{ CqG053 } & 2 & $1 \mathrm{a}, 1 \mathrm{~b}$ & 66 & $\mathrm{n}(\mathrm{h})$ & GQ286117.1 & $\begin{array}{ll}\begin{array}{l}\text { Clone GI_2D } \\
\text { sequences }\end{array} & \text { mRNA } \\
\end{array}$ & $\begin{array}{l}\text { Cherax } \\
\text { quadricarinatus }\end{array}$ & $4.00 \mathrm{E}-21$ & $97(63 / 65)$ \\
\hline & & & & & & $\begin{array}{l}\text { Other nucleotides } \\
\text { sequences }\end{array}$ & & & \\
\hline CqG040 & 1 & $3 c$ & 229 & $\mathrm{n}(\mathrm{s})$ & AM439566.1 & $\begin{array}{lr}\begin{array}{l}\text { Whole } \\
\text { shotgun }\end{array} & \text { genome } \\
\text { sequence } \\
\text { contig VV78X26936.8 }\end{array}$ & Vitis vinifera & $4.00 \mathrm{E}-05$ & $83(48 / 58)$ \\
\hline CqG059 & 1 & $3 a$ & 255 & $\mathrm{n}(\mathrm{s})$ & HM020387.1 & $\begin{array}{lr}\text { Secretory } & \text { eggshell } \\
\text { protein } & \text { precursor } \\
\text { (SEP18.7) mRNA }\end{array}$ & $\begin{array}{l}\text { Clonorchis } \\
\text { sinensis }\end{array}$ & $3.00 \mathrm{E}-20$ & $79(93 / 117)$ \\
\hline
\end{tabular}

When no homology was found with a BLASTx $[\mathrm{x}]$ against non-redundant sequences in the public database, BLASTn optimised for highly similar sequences (megablast) [n (h)] was conducted against sequences in database. If no similarity obtained from $\mathrm{n}(\mathrm{h})$, then BLASTn optimised for somewhat similar sequences (blastn) [n (s)] was performed. If multiple significant similarities matched with a single cDNA (sequence consensus), only the highest scoring hit was included in the table. Library 1a and $1 \mathrm{~b}$ were produced from the first SHH trial with amplicon size of $270 \mathrm{bp}$ and $200 \mathrm{bp}$, respectively. Library 2a was constructed from the second SSH trial with amplicon size of $200 \mathrm{bp}$. Library 3a, 3b and $3 \mathrm{c}$ were constructed from the third SSH trial with amplicon size of $450 \mathrm{bp}, 350 \mathrm{bp}$, and 300 bp respectively. All these bands (amplicons) were cut, purified and cloned to construct the libraries. Library 1, 2 and 3 were constructed from the first, second and third SSH trial respectively, directly purified and inserted to the cloning vector without cutting the bands. $\mathrm{q} / \mathrm{s}$ : number of identical amino acids (nucleotides) between query and subjects sequences/number of amino acids (nucleotides) for alignment. A similarity was considered significant at E-value $<1 \mathrm{e}-05$.

\section{Discussion}

In the present study gills were selected as a target tissue because of its distinct histopathological features between the hypertrophied nuclei and non-hypertrophied nuclei populations. The affected crayfish had hypertrophic nuclei with rarefied chromatin but without CA intranuclear inclusion bodies in the gill epithelium which resembled histological changes consistent with a putative gill parvovirus reported by Edgerton et al. (2000) in this species from northern Queensland. On this basis, it was assumed that these animals were infected with putative gill parvovirus. In addition, as a multifunctional organ, gills not only play an important role in respiration, osmotic and ionic regulation, and detoxification (ClaveroSalas et al., 2007; Freire et al., 2008), but are also considered to be involved in the immune response to invading pathogens (ClaveroSalas et al., 2007; Somboonwiwat et al., 2008; Yeh et al., 2007) and were therefore ideal for detecting up-regulation of immune-related genes using suppression subtractive hybridization.

Many transcripts were identified in the SSH libraries, but for the purpose of this study, only immune-related transcripts will be discussed. Among a variety of transcripts related to immune response, three antimicrobial peptides belonging to lysozyme, crustin and the antilipopolysaccharide (ALF) families were detected. Lysozymes have the ability to lyse bacteria by splitting the glycosidic linkage between N-acetylglucosamine and $\mathrm{N}$ acetylmuramic acid of peptidoglycan in the bacterial cell wall (Bachali et al., 2002; Jolles and Jolles, 1984). Bacteriolytic activity of these enzymes in crustacea has been reported against both Gram-positive and negative bacteria including pathogenic Vibrio species (Burge et al., 2007; Fenouil and Roch, 1991; Hikima et al., 2003; Yao et al., 2008). Lysozymes are also thought to play a role in 
an antiviral response in crustacea (He et al., 2005; Mai and Wang, 2010; Pan et al., 2005). Senapin and Phongdara (2006) found that lysozymes could bind to viral capsid proteins (VP1 and VP2) of Taura syndrome virus (TSV). Similarly, crustin, a cystein-rich peptide that contains a whey acidic protein (WAP) domain also has antibacterial activity against Gram-positive bacteria, but some type II and III crustins have the ability to respond to both Gram-positive and negative bacteria (Donpudsa et al., 2010b). Type III crustins may also have proteinase inhibitory activities (Amparyup et al., 2008).

Like lysozymes, anti-lipopolysaccharide factor can be found across a variety of organisms and has multiple biological activities. This molecule inhibits both Grampositive and negative bacteria and fungus (de la Vega et al., 2008; Sun et al., 2011; Yedery and Reddy, 2009). This antimicrobial peptide is also predicted to have an opsonising function for haemocytes in phagocytosing bacteria (Sun et al., 2011). The ALF may have an immunological function against viral infections in crustacea (Antony et al., 2011; de la Vega et al., 2008; Liu et al., 2011; Liu et al., 2006). It was found that the ALF was upregulated in the WSSV-experimentally infected crayfish and silencing this protein enhanced viral propagation (Liu et al., 2011; Liu et al., 2006). Furthermore, this peptide is considered a potential therapeutic agent for prophylactic treatment of viral and bacterial infectious diseases and septic shock (Somboonwiwat et al., 2008)

Kazal-type serine proteinase inhibitor of Procambarus clarkii and serum amyloid A of Equus caballus transcripts dominated the immune-related genes from the SHH library. The Kazal-type serine proteinase inhibitors are believed to have a role in regulation of immune reactions of crustacea, inhibition of proteinase from microorganisms, bacteriostatic activities against both Grampositive and negative bacteria (Donpudsa et al., 2009; Li et al., 2009) and are probably involved in an antiviral response as well (Donpudsa et al., 2010a; Liu et al., 2011). Serum amyloid A (SAA) is an acute phase protein (APP) that has a role in inflammatory processes in vertebrates. This acute phase protein increased in viral and bacterial infected animals (Cray et al., 2009). Its role in invertebrates, in particular crustaceans, has hardly been investigated. However, in the sea cucumber Holothuria glaberrima, the serum amyloid A was predicted to be involved in intestinal morphogenesis (Santiago-Cardona et al., 2003).

Two transcripts representing translationally controlled tumour proteins (TCPT), also called fortilins, were identified in the SSH libararies. Translationally controlled tumour proteins have been implicated in cell cycle progression, malignant transformation, antiapoptotic activity and cell stress (Bommer and Thiele, 2004). In the banana prawn, Penaeus merguiensis, fortilin was suggested to be involved in early oocyte maturation and may be related to cell proliferation and differentiation (Loongyai et al., 2007). This protein also has binding ability to calcium, tubulin, myeloid cell leukaemia (MCL)-1 protein, elongation factor (EF)-1 $\alpha$ (Bangrak et al., 2004; Bommer and Thiele, 2004; Loongyai et al., 2007) and some transcription factors (Chen et al., 2009). In P. monodon, TCTP was suggested to protect virally infected cells from dying, thus keeping the prawns healthy (Bangrak et al., 2004; Graidist et al., 2006). TCTP could also inhibit viral replication, thus decreasing the amount of viral infection (Tonganunt et al., 2008).

Additional transcripts related to immune factors found in the gill cDNA SSH libraries were chaperonin 10 and eukaryotic initiation factor (eIF) 4A. Chaperonin is a protein that plays an essential role in mediating folding of unfolded polypeptides such as newly translated, imported and stress-denatured proteins. The type of chaperonin determines the process of protein-folding activity. The protein-folding activity of chaperonin I is related to the interaction of chaperonin 60 and chaperonin 10 activities. The type I chaperonin can be found in the chloroplast, eubacteria and mitochondria. Type II chaperonin has only chaperonin 60 and can be found in Archaebacteria and eukaryotic cytosol (Levy-Rimler et al., 2002; Valpuesta et al., 2002). Chaperonin also plays an important role in cellular functions. For example, deletion of mitochondrial yeast and bacterial chaperonins can be lethal to both organisms. It is suggested that in humans hereditary spastic paraplegia spg 13 occurs due to mutation of mitochondrial chaperonin 
60 (Levy-Rimler et al., 2002). Accumulation of toxic protein aggregating in systemic and neurological diseases of humans such as Parkinson's and Huntington's may be related to protein misfolding (Spiess et al., 2004). Chaperonin 10 was also implicated as growth and differentiation factors and may have immunosuppressive activity such as an antiinflammatory activity (Dobocan et al., 2009).

A transcript similar to a matrix cellular protein, putative elastin A of Rimicaris exoculata was also identified in the gill cDNA library. In vertebrates, the presence of elastic fibres in the extracellular space of the connective tissue determines their resilience and maintains pressure related to liquid and air flow. This protein can be found abundantly in the skin, ligament, cartilage, lungs and vascular tissue (Duca et al., 2004; Foster, 2004; Muiznieks et al., 2010). Accordingly, the extracellular matrix content of various organs also determines the susceptibility of the organs to tumour progression. In an experimental tumour model, elastin has been implicated as a factor involved in inhibition of the metastatic processes (Lapis and Tímár, 2002). This protein also has the ability to induce motility signals in cancer cells (Lapis and Tímár, 2002). Despite their biological activities related to cancer, elastin peptides are also suggested to be involved in vasorelaxation, stimulation of leukocytes' oxidative burst, release of lysosomal enzyme, synthesis of endogenous cholesterol, modification of ion fluxes and inducing apoptosis (Duca et al., 2004).

In addition, a gene representing laminin receptor was also expressed in the SSH libraries. Laminin receptor is a protein with a molecular mass about $67 \mathrm{kDa}$ that has high affinity and specificity for laminin (Nelson et al., 2008). The binding of laminin protein to laminin receptor have been implicated in many biological activities such as cell adhesion, proliferation, differentiation and migration. This receptor has also binding ability to elastin and its degradation products (Fülöp and Larbi, 2002). Elastin-laminin receptor plays an important role in extracellular matrix remodelling in aging, atherosclerosis, extravasations, tumour invasion and metastasis (Fülöp and Larbi, 2002; Kunecki and Nawrocka, 2001). In addition, laminin receptor may contribute in bacterial and viral infection (Fülöp and Larbi, 2002; Senapin and Phongdara, 2006). In penaeid prawns, laminin receptor was observed to bind to viral protein (VP) of Taura syndrome virus (TSV), yellow head virus (YHV) and infectious myonecrosis virus (IMNV) (Busayarat et al., 2011; Senapin and Phongdara, 2006). Up-regulation of laminin receptor was found in WSSV-infected redclaw crayfish (Liu et al., 2011), suggesting this receptor has protective function against viral infections in decapod crustacea through binding to viral enveloped proteins that prevents viruses binding to target host cells (Busayarat et al., 2011; Liu et al., 2011).

An antioxidant enzyme, cytosolic manganese superoxide dismutase was detected in the library. This enzyme has been implicated in the immune response of crustacea. The principal function of SOD is to protect host cells against the cytotoxic effect of reactive oxygen species (ROS) produced during the activation of host NADPH-oxidase in the phagocytosis process (Li et al., 2010; Lin et al., 2010). Marchand et al. (2009) found that cMnSOD mRNA expression in hydrothermal crab species, Bythograea thermydron and Segonzacia mesantlantica was significantly higher than in coastal crab species, Necora puber and Cancer pagurus. These authors suggested that the environmental conditions of the hydrothermal vent might induce the cMnSOD expression in the crabs as an adaptive response to the higher exposure to oxidative stress compared to less exposure of littoral crabs.

Finally, within the group of genes related to immune factors, insulin growth factor binding protein (IGFBP) 7 was detected in the SSH library. The IGFBP is a family of secreted proteins that bind to insulin-like growth factor (IGF)-I and -II with high affinity and determines their biological activities (Clemmons, 1997). This protein is involved in IGFs transport, protects them from degradation, limits their binding to receptors and maintains a reservoir of biologically inactive IGFs (Castellanos et al., 2008). Insulin-like growth factor plays an important role in growth and differentiation of normal and malignant cells (Hwa et al., 1999; Navarro et al., 1999). The up regulation of IGFBP 7 in WSSV-infected crayfish suggests 
its involvement in anti-viral defence mechanism (Liu et al., 2011).

In spite of the SSH transcripts that were identified in this study, there was no sequence with homology to parvovirus genes or any other viral genes. Similar results were also found in previous studies when penaeid prawns and crayfish were experimentally infected with WSSV (James et al., 2010; Liu et al., 2011; Wang et al., 2006; Zeng and Lu, 2009; Zhao et al., 2007). In these studies WSSV genomes could not be profiled in the SHH libraries but differentially expressed genes were. However, in the other studies on penaeids experimentally infected with WSSV (García et al., 2009; Reyes et al., 2007) and YHV (Junkunlo et al., 2010; Prapavorarat et al., 2010) both host genes and viral genes were identified in the SSH libraries. These discrepancies may indicate the variability of the suppression subtractive hybridization method in profiling viral genomes in given samples. This is the first study attempting to profile the viral genome in presumptive naturally infected crustacea using the PCRbased cDNA subtraction method.

The absence of parvovirus genes from SSH libraries may be explained by the following reasons. Firstly, the concentration of viral genes in the tester cDNAs may have been too low to be expressed using the SSH technique. Secondly, the poly (A) tail on the mRNA of the parvovirus may be too short for this SSH technology to be successful. The complementary DNA synthetic primer of this protocol contains four poly (T) at the first $5^{\prime}$ end. Thus, the target gene should also have at least four or more poly (A)s in the tail in order to be amplified using this method. However, studies on parvovirus indicate that this may not be the case (Tattersall et al., 2008). Finally, the virus causing these lesions may not have any poly (A) tail, therefore it could not be expressed in the cDNA SSH libraries. Further studies are necessary to determine which hypothesis is more likely and uncover the cause of the hypertrophied nuclei with marginated chromatin in the gills of $C$. quadricarinatus from the hypertrophied nuclei population.

Despite the absence of viral gene detection and therefore a conclusion of an idiopathic aetiology, one should keep in mind that the health status of these two populations of redclaw crayfish was different; resulting in differentially expressed immune-related genes in the two populations, with some genes being up regulated in hypertrophied nuclei animals. These genes represented antimicrobial peptides, proteinase inhibitors, acute phase protein, insulin growth factor binding protein, protein folding, eukaryotic initiation factor and matrix cellular protein, which are all known to be involved in immune reactions. This study has provided an insight into the host-viral interaction at molecular level. It may contribute to the future research on crustacean immunity into establishing immune-intervention strategy to combat the devastating impact of viral diseases in order to maintain production of crustacean aquaculture.

\section{Acknowledgment}

The authors would like to thank Dr. Jennifer Elliman for reading the manuscript and offering valuable suggestions on bench work and Dr. Kathy La Fauce for technical suggestions. This work was partly supported by the Graduate Research Scheme (GRS) Grant 2011 Faculty of Medicine, Health and Biomolecular Sciences, James Cook University. Rusaini is an awardee of Australian Development Scholarship (ADS) Program from Australian Agency for International Development (AusAID).

\section{References}

Amparyup, P., Donpudsa, S. \& Tassanakajon, A. (2008). "Shrimp Single WAP Domain (SWD)-Containing Protein Exhibits Proteinase Inhibitory and Antimicrobial Activities," Developmental \& Comparative Immunology, 32 (12) 1497-1509.

Anderson, I. G. \& Prior, H. C. (1992). "Baculovirus Infections in the Mud Crab, Scylla Serrata, and a Freshwater Crayfish, Cherax Quadricarinatus, from Australia," Journal of Invertebrate Pathology, 60 (3) 265273.

Antony, S. P., Philip, R., Joseph, V. \& Singh, I. S. B. (2011). "Anti-Lipopolysaccharide Factor and Crustin-III, the Anti-White Spot Virus Peptides in Penaeus Monodon: Control of 
Viral Infection by Up-Regulation," Aquaculture, 319 (1-2) 11-17.

Bachali, S., Jager, M., Hassanin, A., Schoentgen, F., Jolles, P., Fiala-Medioni, A. \& Deutsch, J. S. (2002). "Phylogenetic Analysis of Invertebrate Lysozyme and the Evolution of Lysozyme Activity," Journal of Molecular Evolution, 54 (5) 652-664.

Bangrak, P., Graidist, P., Chotigeat, W. \& Phongdara, A. (2004). "Molecular Cloning and Expression of a Mammalian Homologue of a Translationally Controlled Tumor Protein (TCTP) Gene from Penaeus Monodon Shrimp," Journal of Biotechnology, 108 (3) 219-226.

Baumgartner, W. A., Hawke, J. P., Bowles, K., Varner, P. W. \& Hasson, K. W. (2009). "Primary Diagnosis and Surveillance of White Spot Syndrome Virus in Wild and Farmed Crawfish (Procambarus Clarkii, P. Zonangulus) in Louisiana, USA," Diseases of Aquatic Organisms, 85 (1) 15-22.

Belevich, I., Gorbikova, E., Belevich, N. P., Rauhamäki, V., Wikström, M. \& Verkhovsky, M. I. (2010). "Initiation of the Proton Pump of Cytochrome C Oxidase," Proceeding of National Academy of Sciences, 107 (43) 18469-18474.

Bell, T. A. \& Lightner, D. V. (1988). A Handbook of Normal Penaeid Shrimp Histology, World Aquaculture Society, Baton Rouge, Louisiana, USA.

Bommer, U.- A. \& Thiele, B.- J. (2004). "The Translationally Controlled Tumour Protein (TCTP)," The International Journal of Biochemistry \& Cell Biology, 36 (3) 379-385.

Bowater, R. O., Wingfield, M., Fisk, A., Condon, K. M., Reid, A., Prior, H. \& Kulpa, E. C. (2002). "A Parvo-Like Virus in Cultured Redclaw Crayfish Cherax Quadricarinatus from Queensland, Australia," Diseases of Aquatic Organisms, 50 (2) 79-86.

Burge, E. J., Madigan, D. J., Burnett, L. E. \& Burnett, K. G. (2007). "Lysozyme Gene Expression by Hemocytes of Pacific White Shrimp, Litopenaeus Vannamei, after Injection with Vibrio," Fish and Shellfish Immunology, 22 (4) 327-339.
Busayarat, N., Senapin, S., Tonganunt, M., Phiwsaiya, K., Meemetta, W., Unajak, S., Jitrapakdee, S., Lo, C.- F. \& Phongdara, A. (2011). "Shrimp Laminin Receptor Binds with Capsid Proteins of Two Additional Shrimp RNA Viruses YHV and IMNV," Fish and Shellfish Immunology, 31 (1) 66-72.

Castellanos, M., Jiménez-Vega, F. \& VargasAlbores, F. (2008). "Single IB Domain (SIBD) Protein from Litopenaeus Vannamei, a Novel Member for the IGFBP Family," Comparative Biochemistry and Physiology D, 3 (4) 270-274.

Chen, D., He, N., Lei, K. \& Xu, X. (2009). "Genomic Organization of the Translationally Controlled Tumor Protein (TCTP) Gene from Shrimp Marsupenaeus Japonicus," Molecular Biology Reports, 36 (5) 1135-1140.

Clavero-Salas, A., Sotelo-Mundo, R. R., GollasGalván, T., Hernández-López, J., PeregrinoUriarte, A. B., Muhlia-Almazán, A. \& YepizPlascencia, G. (2007). "Transcriptome Analysis of Gills from the White Shrimp Litopenaeus Vannamei Infected with White Spot Syndrome Virus," Fish and Shellfish Immunology, 23 (2) 459-472.

Clemmons, D. R. (1997). “Insulin-Like Growth Factor Binding Proteins and Their Role in Controlling IGF Actions," Cytokine \& Growth Factor Reviews, 8 (1) 45-62.

Cray, C., Zaias, J. \& Altman, N. H. (2009). "Acute Phase Response in Animals: A Review," Comparative Medicine, 59 (6) 517526.

De la Vega, E., O'Leary, N. A., Shockey, J. E., Robalino, J., Payne, C., Browdy, C. L., Warr, G. W. \& Gross, P. S. (2008). "AntiLipopolysaccharide Factor in Litopenaeus Vannamei (LvALF): A Broad Spectrum Antimicrobial Peptide Essential for Shrimp Immunity against Bacterial and Fungal Infection," Molecular Immunology, 45 (7) 1916-1925.

Dobocan, M. C., Sadvakassova, G. \& Congote, L. F. (2009). "Chaperonin 10 as an EndothelialDerived Differentiation Factor: Role of Glycogen Synthase Kinase-3," Journal of Cellular Physiology, 219 (2) 470-476. 
Donpudsa, S., Ponprateep, S., Prapavorarat, A., Visetnan, S., Tassanakajon, A. \& Rimphanitchayakit, V. (2010a). "A Kazal-Type Serine Proteinase Inhibitor SPIPm2 from the Black Tiger Shrimp Penaeus Monodon is Involved in Antiviral Responses," Developmental \& Comparative Immunology, 34 (10) 1101-1108.

Donpudsa, S., Rimphanitchayakit, V., Tassanakajon, A., Söderhäll, I. \& Söderhäll, K. (2010b). "Characterization of Two Crustin Antimicrobial Peptides from the Freshwater Crayfish Pacifastacus Leniusculus," Journal of Invertebrate Pathology, 104 (3) 234-238.

Donpudsa, S., Tassanakajon, A. \& Rimphanitchayakit, V. (2009). "Domain Inhibitory and Bacteriostatic Activities of the Five-Domain Kazal-Type Serine Proteinase Inhibitor from Black Tiger Shrimp Penaeus Monodon," Developmental \& Comparative Immunology, 33 (4) 481-488.

Duca, L., Floquet, N., Alix, A. J. P., Haye, B. \& Debelle, L. (2004) "Elastin as a Matrikine," Critical Reviews in Oncology/Hematology, 49 (3) 235-244.

Edgerton, B. (1996). “A New Bacilliform Virus in Australian Cherax Destructor (Decapoda: Parastacidae) with Notes on Cherax Quadricarinatus Bacilliform Virus (= Cherax Baculovirus)," Diseases of Aquatic Organisms, 27 (1) 43-52.

Edgerton, B. F. (2004). "Susceptibility of the Australian Freshwater Crayfish Cherax Destructor Albidus to White Spot Syndrome Virus (WSSV)," Diseases of Aquatic Organisms, 59 (3) 187-193.

Edgerton, B. F., Evans, L. H., Stephens, F. J. \& Overstreet, R. M. (2002). "Synopsis of Freshwater Crayfish Diseases and Commensal Organisms," Aquaculture, 206 (12) 57-135.

Edgerton, B. F. \& Owens, L. (1999). "Histopathological Surveys of the Redclaw Freshwater Crayfish, Cherax Quadricarinatus, in Australia," Aquaculture, 180 (1-2) 23-40.

Edgerton, B. F., Webb, R., Anderson, I. G. \& Kulpa, E. C. (2000). "Description of a Presumptive Hepatopancreatic Reovirus, and a Putative Gill Parvovirus, in the Freshwater Crayfish Cherax Quadricarinatus," Diseases of Aquatic Organisms, 41 (2) 83-90.

Edgerton, B. \& Owens, L. (1997). “Age at First Infection of Cherax Quadricarinatus by Cherax Quadricarinatus Bacilliform Virus and Cherax Giardiavirus-Like Virus, and Production of Putative Virus-Free Crayfish," Aquaculture, 152 (1-4) 1-12.

Edgerton, B., Owens, L., Glasson, B. \& De Beer, S. (1994). "Description of a Small dsRNA Virus from Freshwater Crayfish Cherax Quadricarinatus," Diseases of Aquatic Organisms, 18 63-69.

Edgerton, B., Webb, R. \& Wingfield, M. (1997). "A Systemic Parvo-Like Virus in the Freshwater Crayfish Cherax Destructor," Diseases of Aquatic Organisms, 29 (1) 73-78.

Fenouil, E. \& Roch, P. (1991). "Evidence and Characterization of Lysozyme in Six Species of Freshwater Crayfish from Astacidae and Cambaridae Families," Comparative Biochemistry and Physiology B, 99 (1) 43-49.

Foster, J. A. (2004). "Elastin," In J. L. William and M. D. Lane (Eds.), Encyclopedia of Biological Chemistry, Elsevier, New York, USA, pp. 10-12.

Freire, C. A., Onken, H. \& McNamara, J. C. (2008). "A Structure-Function Analysis of Ion Transport in Crustacean Gills and Excretory Organs," Comparative Biochemistry and Physiology A, 151 (3) 272-304.

Fülöp, T. \& Larbi, A. (2002). "Putative Role of $67 \mathrm{kDa}$ Elastin-Laminin Receptor in Tumor Invasion," Seminars in Cancer Biology, 12 (3) 219-229.

García, J. C., Reyes, A., Salazar, M. \& Granja, C. B. (2009). "Differential Gene Expression in White Spot Syndrome Virus (WSSV)-Infected Naïve and Previously Challenged Pacific White Shrimp Penaeus (Litopenaeus) Vannamei," Aquaculture, 289 (3-4) 253-258.

Graidist, P., Fujise, K., Wanna, W., Sritunyalucksana, K. \& Phongdara, A. (2006). "Establishing a Role for Shrimp Fortilin in Preventing Cell Death," Aquaculture, 255 (14) 157-164. 
Hayakijkosol, O. \& Owens, L. (2011). "Investigation into the Pathogenicity of Reovirus to Juvenile Cherax Quadricarinatus," Aquaculture, 316 (1-4) 1-5.

He, N., Qin, Q. \& Xu, X. (2005). “Differential Profile of Genes Expressed in Hemocytes of White Spot Syndrome Virus-Resistant Shrimp (Penaeus Japonicus) by Combining Suppression Subtractive Hybridization and Differential Hybridization," Antiviral Research, 66 (1) 39-45.

Hernández, G., Lalioti, V., Vandekerckhove, J., Sierra, J. M. \& Santarén, J. F. (2004). "Identification and Characterization of the Expression of the Translation Initiation Factor 4A (eIF4A) from Drosophila Melanogaster," Proteamics, 4 (2) 316-326.

Hernández, G. \& Vazquez-Pianzola, P. (2005). "Functional Diversity of the Eukaryotic Translation Initiation Factors Belonging to eIF4 Families," Mechanisms of Development, 122 (7-8) 865-876.

Hikima, S., Hikima, J.- I., Rojtinnakorn, J., Hirono, I. \& Aoki, T. (2003). "Characterization and Function of Kuruma Shrimp Lysozyme Possessing Lytic Activity against Vibrio Species," Gene, 316, 187-195.

Hwa, V., Oh, Y. \& Rosenfeld, R. G. (1999). “The Insulin-Like Growth Factor-Binding Protein (IGFBP) Superfamily," Endocrine Reviews, 20 (6) 761-787.

James, R., Thampuran, N., Lalitha, K. V., Rajan, L. A. \& Joseph, T. C. (2010). "Differential Gene Expression Profile of the Hepatopancreas of White Spot Syndrome Virus Infected Fenneropenaeus Indicus by Suppression Subtractive Hybridization," Fish and Shellfish Immunology, 29 (5) 884-889.

Jankowsky, E. (2011). "RNA Helicases at Work: Binding and Rearranging," Trends in Biochemical Sciences, 36 (1) 19-29.

Jiravanichpaisal, P., Bangyeekhun, E., Soderhall, K. \& Soderhall, I. (2001). "Experimental Infection of White Spot Syndrome Virus in Freshwater Crayfish Pacifastacus Leniusculus," Diseases of Aquatic Organisms, 47 (2) 151-157.
Jolles, P. \& Jolles, J. (1984). "What's New in Lysozyme Research? Always a Model System, Today as Yesterday," Molecular and Cellular Biochemistry, 63 (2) 165-189.

Junkunlo, K., Barvornpinyo, S., Flegel, T. W. \& Sritunyalucksana, K. (2010). "Characterization of Putative Immune Genes in Yellow Head Virus (YHV) Infected Shrimp (Penaeus Vannamei) by Suppression Subtractive Hybridization (SSH) Technique," Proceeding of the 48th Kasetsart University Annual Conference, 3-5 March, 2010. Kasetsart University, Bangkok, Thailand, p. unpaginated.

Kunecki, M. \& Nawrocka, A. (2001). "ElastinLaminin Receptor and Abdominal Aortic Aneurysms. New Subject to Study? A Review," Pathologie Biologie, 49(4), 333-338.

Lapis, K. \& Tímár, J. (2002). "Role of ElastinMatrix Interactions in Tumor Progression," Seminars in Cancer Biology, 12 (3) 209-217.

Levy-Rimler, G., Bell, R. E., Ben-Tal, N. \& Azem, A. (2002). "Type I Chaperonins: Not All are Created Equal," FEBS Letters, 529 (1) 1-5.

Li, J., Chen, P., Liu, P., Gao, B., Wang, Q. \& Li, J. (2010). "The Cytosolic Manganese Superoxide Dismutase cDNA in Swimming Crab Portunus Trituberculatus: Molecular Cloning, Characterization and Expression," Aquaculture, 309 (1-4) 31-37.

Lin, Y.- C., Lee, F.- F., Wu, C.- L. \& Chen, J.- C. (2010). "Molecular Cloning and Characterization of a Cytosolic Manganese Superoxide Dismutase (cytMnSOD) and Mitochondrial Manganese Superoxide Dismutase (mtMnSOD) from the Kuruma Shrimp Marsupenaeus Japonicus," Fish and Shellfish Immunology, 28 (1) 143-150.

Liu, H., Jiravanichpaisal, P., Söderhäll, I., Cerenius, L. \& Söderhäll, K. (2006). "Antilipopolysaccharide Factor Interferes with White Spot Syndrome Virus Replication in Vitro and in Vivo in the Crayfish Pacifastacus Leniusculus," Journal of Virology, 80 (21) 10365-10371.

Liu, H.- P., Chen, R.- Y., Zhang, Q.- X., Peng, H. \& Wang, K.- J. (2011). "Differential Gene Expression Profile from Haematopoietic 
Tissue Stem Cells of Red Claw Crayfish, Cherax Quadricarinatus, in Response to WSSV Infection," Developmental \& Comparative Immunology, 35 (7) 716-724.

Li, X.- C., Wang, X.- W., Wang, Z.- H., Zhao, X.F. \& Wang, J.- X. (2009). "A Three-Domain Kazal-Type Serine Proteinase Inhibitor Exhibiting Domain Inhibitory and Bacteriostatic Activities from Freshwater Crayfish Procambarus Clarkii," Developmental \& Comparative Immunology, 33 (12) 12291238.

Loongyai, W., Phongdara, A. \& Chotigeat, W. (2007). "Cloning and Expression of a TCTP Homolog from the Ovaries of Banana Prawn," Marine Biology, 150 (3) 455-462.

Low, W.- K., Dang, Y., Bhat, S., Romo, D. \& Liu, J. O. (2007). "Substrate-Dependent Targeting of Eukaryotic Translation Initiation Factor 4A by Pateamine A: Negation of Domain-Linker Regulation of Activity," Chemistry \& Biology, 14 (6) 715-727.

Mai, W.- J. \& Wang, W.- N. (2010). "Protection of Blue Shrimp (Litopenaeus Stylirostris) against the White Spot Syndrome Virus (WSSV) When Injected with Shrimp Lysozyme," Fish and Shellfish Immunology, 28 (4) 727-733.

Marchand, J., Leignel, V., Moreau, B. \& Chénais, B. (2009). "Characterization and Sequence Analysis of Manganese Superoxide Dismutases from Brachyura (Crustacea: Decapoda): Hydrothermal Bythograeidae versus Littoral Crabs," Comparative Biochemistry and Physiology B, 153 (2) 191199.

Muiznieks, L. D., Weiss, A. S. \& Keeley, F. W. (2010). "Structural Disorder and Dynamics of Elastin," Biochemistry and Cell Biology, 88 (2) 239-250.

Navarro, I., Leibush, B., Moon, T. W., Plisetskaya, E. M., Baños, N., Méndez, E., Planas, J. V. \& Gutiérrez, J. (1999). "Insulin, Insulin-Like Growth Factor-I (IGF-I) and Glucagon: The Evolution of their Receptors," Comparative Biochemistry and Physiology B, 122 (2) 137-153.
Nelson, J., McFerran, N. V., Pivato, G., Chambers, E., Doherty, C., Steele, D. \& Timson, D. J. (2008). "The $67 \mathrm{kDa}$ laminin Receptor: Structure, Function and Role in Disease," Bioscience Reports, 28 (1) 33-48.

Owens, L. (2011). "The Viral Ecology of Aquatic Crustaceans", In C. J. Hurst (Ed.), Studies in Viral Ecology (Vol. 2), Wiley \& Sons, New York, USA, pp. 175-187.

Owens, L. \& McElnea, C. (2000). "Natural Infection of the Redclaw Crayfish Cherax Quadricarinatus with Presumptive SpawnerIsolated Mortality Virus," Diseases of Aquatic Organisms, 40 (3) 219-223.

Pan, D., He, N., Yang, Z., Liu, H. \& Xu, X. (2005). "Differential Gene Expression Profile in Hepatopancreas of WSSV-Resistant Shrimp (Penaeus Japonicus) by Suppression Subtractive Hybridization," Developmental \& Comparative Immunology, 29 (2) 103-112.

Prapavorarat, A., Pongsomboon, S. \& Tassanakajon, A. (2010). "Identification of Genes Expressed in Response to Yellow Head Virus Infection in the Black Tiger Shrimp, Penaeus Monodon, by Suppression Subtractive Hybridization," Developmental \& Comparative Immunology, 34 (6) 611-617.

Reyes, A., Salazar, M. \& Granja, C. (2007). "Temperature Modifies Gene Expression in Subcuticular Epithelial Cells of White Spot Syndrome Virus-Infected Litopenaeus Vannamei," Developmental \& Comparative Immunology, 31 (1) 23-29.

Santiago-Cardona, P. G., Berríos, C. A., Ramírez, F. \& García-Arrarás, J. E. (2003). "Lipopolysaccharides Induce Intestinal Serum Amyloid A Expression in the Sea Cucumber Holothuria Glaberrima," Developmental \& Comparative Immunology, 27 (2) 105-110.

Senapin, S. \& Phongdara, A. (2006). "Binding of Shrimp Cellular Proteins to Taura Syndrome Viral Capsid Proteins VP1, VP2 and VP3," Virus Research, 122 (1-2) 69-77.

Somboonwiwat, K., Bachère, E., Rimphanitchayakit, V. \& Tassanakajon, A. (2008). "Localization of AntiLipopolysaccharide Factor (ALFPm3) in Tissues of the Black Tiger Shrimp, Penaeus 
Monodon, and Characterization of its Binding Properties," Developmental \& Comparative Immunology, 32 (10) 1170-1176.

Spiess, C., Meyer, A. S., Reissmann, S. \& Frydman, J. (2004). "Mechanism of the Eukaryotic Chaperonin: Protein Folding in the Chamber of Secrets," Trends in Cell Biology, 14 (11) 598-604.

Sun, C., Xu, W.- T., Zhang, H.- W., Dong, L.- P., Zhang, T., Zhao, X.- F. \& Wang, J.- X. (2011). "An Anti-Lipopolysaccharide Factor from Red Swamp Crayfish, Procambarus Clarkii, Exhibited Antimicrobial Activities in Vitro and in Vivo," Fish and Shellfish Immunology, 30 (1) 295-303.

Surakasi, V. P. \& Kim, Y. (2010). “Cloning and Expression Pattern of Eukaryotic Translation Initiation Factor 4A of the Diamondback Moth, Plutella Xylostella," Journal of AsiaPacific Entomology, 13 (4) 297-301.

Tattersall, P., Mahy, B. W. J. \& Regenmortel, M. H. V. V. (2008). "Parvoviruses: General Features," Encyclopedia of Virology, Academic Press, Oxford, pp. 90-97.

Tonganunt, M., Nupan, B., Saengsakda, M., Suklour, S., Wanna, W., Senapin, S., Chotigeat, W. \& Phongdara, A. (2008). "The Role of PmFortilin in Protecting Shrimp from White Spot Syndrome Virus (WSSV) Infection," Fish and Shellfish Immunol, 25 (5) 633-637.

Valpuesta, J. M., MartIn-Benito, J., GómezPuertas, P., Carrascosa, J. L. \& Willison, K. R. (2002). "Structure and Function of a Protein Folding Machine: The Eukaryotic Cytosolic Chaperonin CCT," FEBS Letters, 529 (1) 1116.

Wagner, K., Rehling, P., Sanjuán Szklarz, L. K., Taylor, R. D., Pfanner, N. \& van der Laan, M. (2009). "Mitochondrial F1Fo-ATP Synthase: The Small Subunits e and G Associate with Monomeric Complexes to Trigger Dimerization," Journal of Molecular Biology, 392 (4) 855-861.

Wang, B., Li, F., Dong, B., Zhang, X., Zhang, C. \& Xiang, J. (2006). "Discovery of the Genes in Response to white Spot Syndrome Virus (WSSV) Infection in Fenneropenaeus
Chinensis through cDNA Microarray," Marine Biotechnology, 8 (5) 491-500.

Yao, C.- L., Wu, C.- G., Xiang, J.- H., Li, F., Wang, Z.- Y. \& Han, X. (2008). "The Lysosome and Lysozyme Response in Chinese Shrimp Fenneropenaeus Chinensis to Vibrio Anguillarum and Laminarin Stimulation," Journal of Experimental Marine Biology and Ecology, 363 (1-2) 124-129.

Yedery, R. D. \& Reddy, K. V. R. (2009). "Identification, Cloning, Characterization and Recombinant Expression of an AntiLipopolysaccharide Factor from the Hemocytes of Indian Mud Crab, Scylla Serrata," Fish and Shellfish Immunology, 27 (2) 275-284.

Yeh, M.- S., Huang, C.- J., Cheng, J.- H. \& Tsai, I.H. (2007). "Tissue-Specific Expression and Regulation of the Haemolymph Clottable Protein of Tiger Shrimp (Penaeus Monodon)," Fish and Shellfish Immunology, 23 (2) 272279.

Zeng, Y. \& Lu, C.- P. (2009). "Identification of Differentially Expressed Genes in Haemocytes of the Crayfish (Procambarus Clarkii) Infected with White Spot Syndrome Virus by Suppression Subtractive Hybridization and cDNA Microarrays," Fish and Shellfish Immunology, 26 (4) 646-650.

Zhao, Z.- Y., Yin, Z.- X., Weng, S.- P., Guan, H.- J., Li, S.- D., Xing, K., Chan, S.- M. \& He, J.- G. (2007). "Profiling of Differentially Expressed Genes in Hepatopancreas of White Spot Syndrome Virus-Resistant Shrimp (Litopenaeus Vannamei) by Suppression Subtractive Hybridisation," Fish and Shellfish Immunology, 22 (5) 520-534. 